\title{
Spectral Properties of Unbounded Jacobi Matrices with Almost Monotonic Weights
}

\section{Grzegorz Świderski ${ }^{1}$}

Received: 10 December 2014 / Revised: 8 July 2015 / Accepted: 30 July 2015 /

Published online: 8 November 2015

(C) The Author(s) 2015. This article is published with open access at Springerlink.com

\begin{abstract}
We present a unified framework to identify spectra of Jacobi matrices. We give applications of the long-standing problem of Chihara (Mt J Math 21(1):121-137, 1991, J Comput Appl Math 153(1-2):535-536, 2003) concerning one-quarter class of orthogonal polynomials, to the conjecture posed by Roehner and Valent (SIAM J Appl Math 42(5):1020-1046, 1982) concerning continuous spectra of generators of birth and death processes, and to spectral properties of operators studied by Janas and Moszyńki (Integral Equ Oper Theory 43(4):397-416, 2002) and Pedersen (Proc Am Math Soc 130(8):2369-2376, 2002).
\end{abstract}

Keywords Jacobi matrix · Continuous spectrum · One-quarter class of orthogonal polynomials

Mathematics Subject Classification Primary: 47B36; Secondary: 42C05 · 60J80

\section{Introduction}

Given sequences $\left\{a_{n}\right\}_{n=0}^{\infty}$ and $\left\{b_{n}\right\}_{n=0}^{\infty}$ such that $a_{n}>0$ and $b_{n} \in \mathbb{R}$, we set

$$
A=\left(\begin{array}{cccccc}
b_{0} & a_{0} & 0 & 0 & 0 & \ldots \\
a_{0} & b_{1} & a_{1} & 0 & 0 & \ldots \\
0 & a_{1} & b_{2} & a_{2} & 0 & \ldots \\
0 & 0 & a_{2} & b_{3} & a_{3} & \ldots \\
\vdots & \vdots & \vdots & \vdots & \vdots & \ddots
\end{array}\right) .
$$

Communicated by Doron S. Lubinsky.

Grzegorz Świderski

gswider@math.uni.wroc.pl

1 Instytut Matematyczny, Uniwersytet Wrocławski, Pl. Grunwaldzki 2/4, 50-384 Wrocław, Poland 
The operator $A$ is defined on the domain $\operatorname{Dom}(A)=\left\{x \in \ell^{2}: A x \in \ell^{2}\right\}$, where

$$
\ell^{2}=\left\{x \in \mathbb{C}^{\mathbb{N}}: \sum_{n=0}^{\infty}\left|x_{n}\right|^{2}<\infty\right\},
$$

and is called a Jacobi matrix.

The study of Jacobi matrices is motivated by connections with orthogonal polynomials and the classical moment problem (see, e.g., [23]). Also, every self-adjoint operator can be represented as a direct sum of Jacobi matrices. In particular, generators of birth and death processes may be seen as Jacobi matrices acting on weighted $\ell^{2}$ spaces.

There are several approaches to the problem of the identification of the spectrum of unbounded Jacobi matrices. A method often used is based on subordination theory (see, e.g., $[6,15,19]$ ). Another technique uses the analysis of a commutator between a Jacobi matrix and a suitable chosen matrix (see, e.g., [22]). The case of Jacobi matrices with monotonic weights was considered mainly by Dombrowski (see, e.g., [8]), where the author developed commutator techniques which enabled qualitative spectral analysis of examined operators.

The present article is motivated by commutator techniques of Dombrowski and some ideas of Clark [6]. In fact, commutators do not appear here directly but are hidden in some of our expressions.

Let $A$ be a Jacobi matrix, and assume that the matrix $A$ is self-adjoint. The spectrum of the operator $A$ will be denoted by $\sigma(A)$, the set of all its eigenvalues by $\sigma_{\mathrm{p}}(A)$, and the set of all accumulation points of $\sigma(A)$ by $\sigma_{\text {ess }}(A)$. For a real number $x$, we define $x^{-}=\max (-x, 0)$.

Our main result is the following theorem.

Theorem 1.1 Let A be a Jacobi matrix. If there is a positive sequence $\left\{\alpha_{n}\right\}$ such that

(a) $\lim _{n \rightarrow \infty} a_{n}=\infty$,

(b) $\sum_{n=1}^{\infty}\left[\frac{a_{n+1}}{a_{n}} \frac{\alpha_{n+1}}{\alpha_{n}}-\frac{a_{n}}{a_{n-1}} \frac{\alpha_{n-1}}{\alpha_{n}}\right]^{-}<\infty$,

(c) $\sum_{n=1}^{\infty} \frac{1}{a_{n-1}}\left|\frac{a_{n-1}}{a_{n}}-\frac{\alpha_{n-1}}{\alpha_{n}}\right|<\infty$,

(d) $\sum_{n=1}^{\infty}\left|\frac{b_{n+1}}{a_{n}}-\frac{b_{n}}{a_{n-1}} \frac{\alpha_{n-1}}{\alpha_{n}}\right|<\infty$,

(e) $\sum_{n=0}^{\infty} \frac{1}{a_{n} \alpha_{n}}=\infty$,

(f) $\lim _{n \rightarrow \infty} \frac{\alpha_{n-1}}{\alpha_{n}} \frac{a_{n}}{a_{n-1}}=1$,

(g) $\limsup _{n \rightarrow \infty} \frac{\left|b_{n}\right|}{a_{n}}<2$,

then the Jacobi matrix $A$ is self-adjoint and satisfies $\sigma_{\mathrm{p}}(A)=\emptyset$, and $\sigma(A)=\mathbb{R}$. 
The importance of Theorem 1.1 lies in the fact that we have flexibility in the choice of the sequence $\alpha_{n}$. Some choices of the sequence $\alpha_{n}$ are given in Sect. 4 .

Let us concentrate now on the simplest case of the theorem, i.e., when $\alpha_{n}=a_{n}$. In [11, Lemma 2.6], it was proven that if the nonnegative sequence $a_{n}^{2}-a_{n-1}^{2}$ is bounded and $b_{n} \equiv 0$, then the matrix $A$ has no eigenvalues. Our theorem gives additional information that in this case $\sigma(A)=\mathbb{R}$ holds. Moreover, our assumptions are weaker than the conditions of [11, Lemma 2.6].

In Sect. 6, we provide examples showing sharpness of the assumptions in the case $\alpha_{n}=a_{n}$. In particular, condition (e) is necessary in the class of monotonic sequences $\left\{a_{n}\right\}$, and condition (b) could not be replaced by $\left[\left(a_{n+1} / a_{n}\right)^{2}-1\right]^{-} \rightarrow 0$. Corollary 1.3 shows that in general, condition $(\mathrm{g})$ is necessary.

In Sect. 5, we apply the theorem for $\alpha_{n}=a_{n}$ to resolve a conjecture (see [21]) about continuous spectra of generators of birth and death processes. We also present there applications to the following problem.

Problem 1.2 (Chihara [4,5]) Assume that a Jacobi matrix $A$ is self-adjoint, $b_{n} \rightarrow \infty$, the smallest point $\rho$ of $\sigma_{\text {ess }}(A)$ is finite, and

$$
\lim _{n \rightarrow \infty} \frac{a_{n}^{2}}{b_{n} b_{n+1}}=\frac{1}{4} .
$$

Find additional assumptions (if needed) to assure that $\sigma_{\mathrm{ess}}(A)=[\rho, \infty)$.

A direct consequence of the theorem in the case $\alpha_{n}=a_{n}$, providing easy to check additional assumptions to Problem 1.2, is the following result.

\section{Corollary 1.3 Assume}

(a) $\lim _{n \rightarrow \infty} a_{n}=\infty$

(b) $\sum_{n=0}^{\infty} \frac{1}{a_{n}}=\infty$,

(c) $\sum_{n=0}^{\infty}\left[\frac{a_{n+1}}{a_{n}}-1\right]^{-}<\infty$,

(d) $\lim _{n \rightarrow \infty}\left[a_{n-1}-b_{n}+a_{n}\right]=M$.

Then the Jacobi matrix A satisfies $\sigma_{\mathrm{ess}}(A)=[-M, \infty)$. Moreover, if $a_{n+1} / a_{n} \rightarrow 1$, then

$$
\lim _{n \rightarrow \infty} \frac{a_{n}^{2}}{b_{n} b_{n+1}}=\frac{1}{4} .
$$

Let us present ideas behind the proof of the theorem. Let the difference operator $J$ be defined by

$$
(J x)_{n}=-\alpha_{n-1} x_{n-1}+\alpha_{n} x_{n+1} \quad(n \geq 0)
$$


for a positive sequence $\left\{\alpha_{n}\right\}_{n=0}^{\infty}$ and $\alpha_{-1}=x_{-1}=0$. Then we define commutator $K$ on finite sequences by the formula

$$
2 K=J A-A J .
$$

The expression $S_{n}=\left\langle K\left(p^{n}\right), p^{n}\right\rangle$, where $p^{n}=\left(p_{0}, p_{1}, \ldots, p_{n}, 0,0, \ldots\right),\left\{p_{k}\right\}$ is the formal eigenvector of $A$, and $\langle\cdot, \cdot\rangle$ is the scalar product on $\ell^{2}$, proved to be a useful tool to show that the matrix $A$ has continuous spectrum (see, e.g., $[8,11,13]$ ).

An important observation is that we can give closed form for $S_{n}$ [see (3.1)]. To the author's knowledge, this closed form has been known only for $\alpha_{n}=a_{n}$ (see [7]). A related expression for $\alpha_{n} \equiv 1$ was analyzed in [6]. Adaptation of techniques from [6] allow us to circumvent technical difficulties present in Dombrowski's approach. Extending the definition of $S_{n}$ to generalized eigenvectors [see (2.1)] enables us to show that $\sigma(A)=\mathbb{R}$.

The article is organized as follows. In Sect. 2, we present definitions and wellknown facts important for our argument. In Sect. 3, we prove Theorem 1.1, whereas in Sect. 4 we show its variants. In particular, we identify spectra of operators considered in [20] and [14]. In Sect. 5, we present applications of our theorem to some open problems. Finally, in the last section, we discuss the necessity of the assumptions in the case $\alpha_{n}=a_{n}$. We also present examples showing that in some cases, our results are stronger than results known in the literature.

\section{Tools}

Given a Jacobi matrix $A, \lambda \in \mathbb{R}$ and real numbers $(a, b) \neq(0,0)$, we introduce a generalized eigenvector $u$ by

$$
\begin{aligned}
& u_{0}=a, \quad u_{1}=b, \\
& a_{n} u_{n+1}=\left(\lambda-b_{n}\right) u_{n}-a_{n-1} u_{n-1} \quad(n \geq 1) .
\end{aligned}
$$

Furthermore, we define the sequence

$$
\begin{aligned}
& p_{-1}(\lambda)=0, \quad p_{0}(\lambda)=1, \\
& a_{n} p_{n+1}(\lambda)=\left(\lambda-b_{n}\right) p_{n}(\lambda)-a_{n-1} p_{n-1}(\lambda) \quad(n \geq 0) .
\end{aligned}
$$

The sequence $\left\{p_{n}(\lambda)\right\}$ is a formal eigenvector of matrix $A$ associated with an eigenvalue $\lambda$.

Observe that $\left\{p_{n}(\cdot)\right\}_{n=0}^{\infty}$ is a sequence of polynomials. Moreover, the sequence is orthonormal with respect to the measure $\mu(\cdot)=\left\langle E(\cdot) \delta_{0}, \delta_{0}\right\rangle$, where $E$ is the spectral resolution of the matrix $A,\langle\cdot, \cdot\rangle$ is the scalar product on $\ell^{2}$, and $\delta_{0}=(1,0,0, \ldots)$.

The following propositions are well known. We include them for the sake of completeness.

Proposition 2.1 Let $\lambda \in \mathbb{R}$. If every generalized eigenvector $u$ does not belong to $\ell^{2}$, then the matrix $A$ is self-adjoint, $\lambda \notin \sigma_{\mathrm{p}}(A)$, and $\lambda \in \sigma(A)$. 
Proof [23, Theorem 3] asserts that $A$ is self-adjoint provided that at least one generalized eigenvector $\left\{u_{n}\right\} \notin \ell^{2}$. Direct computation shows that $\lambda \in \sigma_{\mathrm{p}}(A)$ if and only if $\left\{p_{n}(\lambda)\right\} \in \ell^{2}$. Therefore the matrix $A$ is self-adjoint and $\lambda \notin \sigma_{\mathrm{p}}(A)$.

Observe that the vector $x$ such that $(A-\lambda I) x=\delta_{0}$ satisfies the following recurrence relation:

$$
\begin{aligned}
& b_{0} x_{0}+a_{0} x_{1}=\lambda x_{0}+1, \\
& a_{n-1} x_{n-1}+b_{n} x_{n}+a_{n} x_{n+1}=\lambda x_{n} \quad(n \geq 1) .
\end{aligned}
$$

Hence $x$ is a generalized eigenvector, thus $x \notin \ell^{2}$. Therefore, the operator $A-\lambda I$ is not surjective, i.e., $\lambda \in \sigma(A)$.

The following proposition is well known. For the proof we refer to, e.g., [12, Corollary 3.6].

Proposition 2.2 Let $A$ and $\widehat{A}$ be Jacobi matrices defined by sequences $\left\{a_{n}\right\},\left\{b_{n}\right\}$ and $\left\{a_{n}\right\},\left\{-b_{n}\right\}$, respectively. Then

$$
\sigma(A)=-\sigma(\widehat{A}), \quad \sigma_{\mathrm{p}}(A)=-\sigma_{\mathrm{p}}(\widehat{A}) .
$$

The following proposition has been used many times in the literature (see, e.g., $[11,12])$.

Proposition 2.3 Let $A$ be a self-adjoint Jacobi matrix associated with the sequence $b_{n} \equiv 0$. Let $A_{e}$ and $A_{o}$ be restrictions of $A \cdot A$ to the subspaces $\operatorname{span}\left\{\delta_{2 k}: k \in \mathbb{N}\right\}$ and $\operatorname{span}\left\{\delta_{2 k+1}: k \in \mathbb{N}\right\}$, respectively. Then $A_{e}$ and $A_{o}$ are Jacobi matrices associated with

$$
\begin{aligned}
& a_{n}^{e}=a_{2 n} a_{2 n+1}, \quad b_{n}^{e}=a_{2 n-1}^{2}+a_{2 n}^{2}, \\
& a_{n}^{o}=a_{2 n+1} a_{2 n+2}, \quad b_{n}^{o}=a_{2 n}^{2}+a_{2 n+1}^{2},
\end{aligned}
$$

respectively. Moreover, $A_{o}$ and $A_{e}$ are self-adjoint, and

$$
\sigma\left(A_{o}\right)=\sigma\left(A_{e}\right)=(\sigma(A))^{2}, \quad \sigma_{\mathrm{p}}\left(A_{o}\right)=\sigma_{\mathrm{p}}\left(A_{e}\right)=\left(\sigma_{\mathrm{p}}(A)\right)^{2}
$$

when $0 \notin \sigma_{\mathrm{p}}(A)$ and $0 \notin \sigma_{\mathrm{p}}(\widetilde{A})$, where $\widetilde{A}$ is a self-adjoint Jacobi matrix associated with the sequences $\left\{a_{n+1}\right\}_{n=0}^{\infty}$ and $b_{n} \equiv 0$, and for a set $X$, we define $X^{2}=\left\{x^{2}: x \in X\right\}$.

Proof By direct computation it may be proved that $A_{o}$ and $A_{e}$ satisfy (2.2).

Let $\left\{p_{n}^{e}\right\}$ be the sequence of polynomials associated with the matrix $A_{e}$. Then [23, Theorem 3] asserts that $A_{e}$ is self-adjoint provided $\left\{p_{n}^{e}(0)\right\} \notin \ell^{2}$. It is known that $p_{2 n}(x)=p_{n}^{e}\left(x^{2}\right)$ (see, e.g., [12, Section 4]). Since $p_{2 k+1}(0)=0$ and $0 \notin \sigma_{\mathrm{p}}(C)$, we have

$$
\infty=\sum_{n=0}^{\infty} p_{n}^{2}(0)=\sum_{n=0}^{\infty} p_{2 n}^{2}(0)=\sum_{n=0}^{\infty}\left(p_{n}^{e}(0)\right)^{2}
$$

Therefore $A_{e}$ is self-adjoint. 
Assume that $0 \notin \sigma_{\mathrm{p}}(\widetilde{A})$. Observe that $A_{o}=\widetilde{A}_{e}$. Therefore, the previous argument applied to $\widetilde{A}$ implies also that $A_{o}$ is self-adjoint.

The conclusion of spectra follows from, e.g., [12, Section 4].

\section{Proof of the Main Theorem}

Given a generalized eigenvector $u$ and a positive sequence $\left\{\alpha_{n}\right\}$, we set

$$
S_{n}=a_{n-1} \alpha_{n-1} u_{n-1}^{2}+a_{n} \alpha_{n} u_{n}^{2}-\left(\lambda-b_{n}\right) \alpha_{n-1} u_{n-1} u_{n} \quad(n \geq 1) .
$$

Using the identity $a_{n-1} u_{n-1}=\left(\lambda-b_{n}\right) u_{n}-a_{n} u_{n+1}$, we get an equivalent formula,

$$
S_{n}=a_{n} \alpha_{n} u_{n}^{2}+\frac{\alpha_{n-1}}{a_{n-1}} a_{n}^{2} u_{n+1}^{2}-\frac{\alpha_{n-1}}{a_{n-1}} a_{n}\left(\lambda-b_{n}\right) u_{n} u_{n+1} \quad(n \geq 1) .
$$

The sequence $S_{n}$ for $\alpha_{n}=a_{n}$ was previously used in the study of Jacobi matrices but only in the case of bounded ones (see, e.g., [7,10]). In the case of unbounded operators, a sequence similar to $S_{n}$ for $\alpha_{n} \equiv 1$ was also used in [6].

The following proposition is an adaptation of [6, Lemma 3.1].

Proposition 3.1 Let $u$ be a generalized eigenvector associated with $\lambda \in \mathbb{R}$ and

$$
\widetilde{S}_{n}=u_{n}^{2}+u_{n+1}^{2} .
$$

Assume that $a_{n} \rightarrow \infty$, and

$$
\lim _{n \rightarrow \infty} \frac{\alpha_{n-1}}{\alpha_{n}} \frac{a_{n}}{a_{n-1}}=1, \quad \limsup _{n \rightarrow \infty} \frac{\left|b_{n}\right|}{a_{n}}<2 .
$$

Then there exist constants $c_{1}>0, c_{2}>0$ such that for sufficiently large $n$,

$$
c_{1} a_{n} \alpha_{n} \leq \frac{S_{n}}{\widetilde{S}_{n}} \leq c_{2} a_{n} \alpha_{n} .
$$

Proof Observe that from the representation (3.2) we have that $S_{n}$ is a quadratic form with respect to variables $u_{n}$ and $u_{n+1}$. Let the minimal and the maximal value of $S_{n}$ under the condition $\widetilde{S}_{n}=1$ be denoted by $w_{n}^{\min }$ and $w_{n}^{\max }$, respectively. Then

$$
\begin{aligned}
& \frac{2 w_{n}^{\min }}{a_{n} \alpha_{n}}=1+\frac{\alpha_{n-1}}{\alpha_{n}} \frac{a_{n}}{a_{n-1}}-\sqrt{\left(1-\frac{\alpha_{n-1}}{\alpha_{n}} \frac{a_{n}}{a_{n-1}}\right)^{2}+\left(\frac{\alpha_{n-1}}{\alpha_{n}} \frac{a_{n}}{a_{n-1}} \frac{\lambda-b_{n}}{a_{n}}\right)^{2}}, \\
& \frac{2 w_{n}^{\max }}{a_{n} \alpha_{n}}=1+\frac{\alpha_{n-1}}{\alpha_{n}} \frac{a_{n}}{a_{n-1}}+\sqrt{\left(1-\frac{\alpha_{n-1}}{\alpha_{n}} \frac{a_{n}}{a_{n-1}}\right)^{2}+\left(\frac{\alpha_{n-1}}{\alpha_{n}} \frac{a_{n}}{a_{n-1}} \frac{\lambda-b_{n}}{a_{n}}\right)^{2}} .
\end{aligned}
$$

Letting $n \rightarrow \infty$, we see that for large $n$, there is a positive upper and lower bound of the above expressions. This completes the proof. 
Corollary 3.2 Under the assumptions of Proposition 3.1, together with

$$
\sum_{n=0}^{\infty} \frac{1}{a_{n} \alpha_{n}}=\infty
$$

if $\liminf _{n} S_{n}>0$, then $u \notin \ell^{2}$.

Proof Since $\liminf { }_{n} S_{n}>0$ by Proposition 3.1, there exists a constant $c>0$ such that for every $n$ sufficiently large we have

$$
\frac{c}{a_{n} \alpha_{n}} \leq \widetilde{S}_{n}
$$

This shows that $u$ cannot belong to $\ell^{2}$.

Now we are ready to prove Theorem 1.1.

Proof (of Theorem 1.1) By virtue of Corollary 3.2, it is sufficient to show that $\liminf _{n} S_{n}>0$ for every generalized eigenvector $\left\{u_{n}\right\}$.

By Proposition 3.1, there exists $N$ such that for every $n \geq N, S_{n}>0$ holds. Let us define $F_{n}=\left(S_{n+1}-S_{n}\right) / S_{n}$. Then $S_{n+1} / S_{n}=1+F_{n}$; thus

$$
\frac{S_{n}}{S_{N}}=\prod_{k=N}^{n-1}\left(1+F_{n}\right)
$$

Hence

$$
\sum_{n=1}^{\infty} F_{n}^{-}<\infty
$$

implies $\lim \inf _{n} S_{n}>0$. Observe that by (3.1) and (3.2), we get

$$
\begin{aligned}
S_{n+1}-S_{n}= & \left(a_{n+1} \alpha_{n+1}-\frac{\alpha_{n-1}}{a_{n-1}} a_{n}^{2}\right) u_{n+1}^{2} \\
& +\left(\frac{\alpha_{n-1}}{a_{n-1}} a_{n}\left(\lambda-b_{n}\right)-\alpha_{n}\left(\lambda-b_{n+1}\right)\right) u_{n} u_{n+1} .
\end{aligned}
$$

Therefore,

$$
\begin{aligned}
F_{n}= & \frac{S_{n+1}-S_{n}}{S_{n}}=\left[\left(a_{n+1} \alpha_{n+1}-\frac{\alpha_{n-1}}{a_{n-1}} a_{n}^{2}\right) \frac{u_{n+1}^{2}}{\widetilde{S}_{n}}\right. \\
& \left.+\left(\frac{\alpha_{n-1}}{a_{n-1}} a_{n}\left(\lambda-b_{n}\right)-\alpha_{n}\left(\lambda-b_{n+1}\right)\right) \frac{u_{n} u_{n+1}}{\widetilde{S}_{n}}\right] \frac{\widetilde{S}_{n}}{S_{n}},
\end{aligned}
$$

where $\widetilde{S}_{n}=u_{n}^{2}+u_{n+1}^{2}$. By Proposition 3.1 and $\left|u_{n} u_{n+1}\right| / \widetilde{S}_{n} \leq 1$, there exists a constant $c>0$ such that

$$
F_{n}^{-} \leq \frac{c}{a_{n} \alpha_{n}}\left(\left[a_{n+1} \alpha_{n+1}-\frac{\alpha_{n-1}}{a_{n-1}} a_{n}^{2}\right]^{-}+\left|\frac{\alpha_{n-1}}{a_{n-1}} a_{n}\left(\lambda-b_{n}\right)-\alpha_{n}\left(\lambda-b_{n+1}\right)\right|\right) .
$$


Since

$$
\frac{1}{a_{n} \alpha_{n}}\left[a_{n+1} \alpha_{n+1}-\frac{\alpha_{n-1}}{a_{n-1}} a_{n}^{2}\right]^{-}=\left[\frac{a_{n+1}}{a_{n}} \frac{\alpha_{n+1}}{\alpha_{n}}-\frac{a_{n}}{a_{n-1}} \frac{\alpha_{n-1}}{\alpha_{n}}\right]^{-}
$$

and

$$
\begin{aligned}
\frac{1}{a_{n} \alpha_{n}}\left|\frac{\alpha_{n-1}}{a_{n-1}} a_{n}\left(\lambda-b_{n}\right)-\alpha_{n}\left(\lambda-b_{n+1}\right)\right| \\
=\left|\lambda\left(\frac{1}{a_{n-1}} \frac{\alpha_{n-1}}{\alpha_{n}}-\frac{1}{a_{n}}\right)+\left(\frac{b_{n+1}}{a_{n}}-\frac{b_{n}}{a_{n-1}} \frac{\alpha_{n-1}}{\alpha_{n}}\right)\right| \\
\quad \leq \frac{|\lambda|}{a_{n-1}}\left|\frac{\alpha_{n-1}}{\alpha_{n}}-\frac{a_{n-1}}{a_{n}}\right|+\left|\frac{b_{n+1}}{a_{n}}-\frac{b_{n}}{a_{n-1}} \frac{\alpha_{n-1}}{\alpha_{n}}\right|,
\end{aligned}
$$

we obtain (3.3).

Remark 3.3 If we replace the condition (b) by

(b') $\sum_{n=0}^{\infty}\left|\frac{a_{n+1}}{a_{n}} \frac{\alpha_{n+1}}{\alpha_{n}}-\frac{a_{n}}{a_{n-1}} \frac{\alpha_{n-1}}{\alpha_{n}}\right|<\infty$,

then $S_{n}$ is in fact convergent to a positive number. This shows that for every generalized eigenvector $u$, there are constants $c_{1}>0, c_{2}>0$ such that $c_{1} /\left(a_{n} \alpha_{n}\right) \leq u_{n}^{2}+u_{n+1}^{2} \leq$ $c_{2} /\left(a_{n} \alpha_{n}\right)$. Hence for every generalized eigenvectors $u, v$ associated with $\lambda \in \mathbb{R}$, there is a constant $c>0$ such that

$$
\limsup _{N \rightarrow \infty} \frac{\sum_{n=0}^{N}\left|u_{n}\right|^{2}}{\sum_{n=0}^{N}\left|v_{n}\right|^{2}} \leq c .
$$

This estimate together with the subordination method (see, e.g., $[6,15])$ shows that the spectrum of the matrix $A$ is purely absolutely continuous.

\section{Special Cases}

In this section, we are going to show a few choices of the sequence $\left\{\alpha_{n}\right\}$ from Theorem 1.1. In this way, we show the flexibility of our approach.

The following theorem was proved in [14, Theorem 1.6] and is a generalization of [6, Theorem 1.10]. In the proof, the authors analyze transfer matrices. Therefore, our argument gives an alternative proof.

Theorem 4.1 (Janas and Moszyński [14] ) Assume

(a) $\lim _{n \rightarrow \infty} a_{n}=\infty$

(b) $\sum_{n=0}^{\infty} \frac{1}{a_{n}}=\infty$, 
(c) the sequences $\left\{\frac{a_{n+1}}{a_{n}}\right\},\left\{\frac{1}{a_{n}}\right\}$ and $\left\{\frac{b_{n}}{a_{n}}\right\}$ are of bounded variation,

(d) $\lim _{n \rightarrow \infty} \frac{\left|b_{n}\right|}{a_{n}}<2$.

Then $\sigma(A)=\mathbb{R}$, and the matrix A has purely absolutely continuous spectrum.

Proof Let $\alpha_{n} \equiv 1$. By virtue of Remark 3.3, we need to check the assumptions (b'), (d), and (f) of Theorem 1.1.

Since the sequence $\left\{a_{n+1} / a_{n}\right\}$ is of bounded variation, it is convergent to a number $a$. From the condition (b), we have $a \leq 1$, whereas the condition (a) gives $a \geq 1$. This proves the conditions (b') and (f) of Theorem 1.1.

The sequence $\left\{b_{n+1} / a_{n}\right\}$ is of bounded variation because $\frac{b_{n+1}}{a_{n}}=\frac{b_{n+1}}{a_{n+1}} \cdot \frac{a_{n+1}}{a_{n}}$. The proof is complete.

The next theorem imposes very simple conditions on Jacobi matrices. In Sect. 5, we show its applications; furthermore, in Sect. 6, we discuss sharpness of the assumptions.

Theorem 4.2 Assume

(a) $\lim _{n \rightarrow \infty} a_{n}=\infty$,

(b) $\sum_{n=0}^{\infty} \frac{1}{a_{n}^{2}}=\infty$,

(c) $\sum_{n=0}^{\infty}\left[\left(\frac{a_{n+1}}{a_{n}}\right)^{2}-1\right]^{-}<\infty$,

(d) $\limsup _{n \rightarrow \infty} \frac{\left|b_{n}\right|}{a_{n}}<2$,

(e) $\sum_{n=0}^{\infty} \frac{\left|b_{n+1}-b_{n}\right|}{a_{n}}<\infty$.

Then the Jacobi matrix $A$ is self-adjoint and satisfies $\sigma_{\mathrm{p}}(A)=\emptyset$ and $\sigma(A)=\mathbb{R}$.

Proof Apply Theorem 1.1 with $\alpha_{n}=a_{n}$.

Special cases of the following theorem were examined in [20] and [14] using commutator methods.

Theorem 4.3 Let $\log ^{(i)}$ be defined by $\log ^{(0)}(x)=x, \log ^{(i+1)}(x)=\log (\log (i)(x))$. Let $g_{j}(n)=\prod_{i=1}^{j} \log ^{(i)}(n)$. Assume that for positive numbers $K, N$ and for a summable nonnegative sequence $c_{n}$,

(a) $\lim _{n \rightarrow \infty} a_{n}=\infty$, 
(b) $1-c_{n} \leq \frac{a_{n}}{a_{n-1}} \leq 1+\frac{1}{n}+\sum_{j=1}^{K} \frac{1}{n g_{j}(n)}+c_{n}$ for $n>N$,

(c) the sequence $\left\{b_{n}\right\}$ is bounded and $\sum_{n=0}^{\infty} \frac{\left|b_{n+1}-b_{n}\right|}{a_{n}}<\infty$,

(d) $\sum_{n=1}^{\infty} \frac{1}{n a_{n}}<\infty$.

Then $\sigma_{\mathrm{p}}(A)=\emptyset$ and $\sigma(A)=\mathbb{R}$.

Proof We can assume that $\log ^{(K)}(N)>0$. Set

$$
\alpha_{n}= \begin{cases}1 & \text { for } n<N \\ \frac{n g_{K}(n)}{a_{n}} & \text { otherwise }\end{cases}
$$

To get the conclusion, we need to check the assumptions (b), (d), and (c) of Theorem 1.1 .

To show Theorem 1.1(b), let us observe that the assumption (b) of the present theorem gives

$$
\left(\frac{a_{n}}{a_{n-1}}\right)^{2} \leq 1+\frac{2}{n}+\sum_{j=1}^{K} \frac{2}{n g_{j}(n)}+c_{n}^{\prime}
$$

for a summable sequence $c_{n}^{\prime}$. Therefore,

$$
\begin{gathered}
\frac{a_{n+1}}{a_{n}} \frac{\alpha_{n+1}}{\alpha_{n}}-\frac{a_{n}}{a_{n-1}} \frac{\alpha_{n-1}}{\alpha_{n}}=\frac{n+1}{n} \frac{g_{K}(n+1)}{g_{K}(n)}-\left(\frac{a_{n}}{a_{n-1}}\right)^{2} \frac{n-1}{n} \frac{g_{K}(n-1)}{g_{K}(n)} \\
\geq \frac{n+1}{n} \frac{g_{K}(n+1)}{g_{K}(n)}-\frac{n-1}{n}\left(1+\frac{2}{n}+\sum_{j=1}^{K} \frac{2}{n g_{j}(n)}+c_{n}^{\prime}\right) \frac{g_{K}(n-1)}{g_{K}(n)} \\
\geq \frac{n+1}{n} \frac{g_{K}(n+1)}{g_{K}(n)}-\left(\frac{n+1}{n}+\sum_{j=1}^{K} \frac{2}{n g_{j}(n)}+c_{n}^{\prime}\right) \frac{g_{K}(n-1)}{g_{K}(n)} .
\end{gathered}
$$

Since the functions $g_{j}$ are increasing, we have

$$
\geq \frac{n-1}{n}\left(\frac{g_{K}(n+1)-g_{K}(n-1)}{g_{K}(n)}\right)-\frac{g_{K}(n-1)}{n g_{K}(n)} \sum_{j=1}^{K} \frac{2}{g_{j}(n-1)}-c_{n}^{\prime} .
$$


Next, observe that

$$
g_{K}^{\prime}(x)=g_{K}(x) \sum_{j=1}^{K} \frac{\left(\log ^{(j)}\right)^{\prime}(x)}{\log ^{(j)}(x)} .
$$

Therefore,

$$
g_{K}^{\prime}(x)=g_{K}(x) \sum_{j=1}^{K} \frac{1}{x g_{j}(x)}
$$

Hence Taylor's formula applied to $g_{K}$ at the point $n-1$ gives

$$
(n-1)\left[g_{K}(n+1)-g_{K}(n-1)\right]=g_{K}(n-1) \sum_{j=1}^{K} \frac{2}{g_{j}(n-1)}+2(n-1) g_{K}^{\prime \prime}(\xi)
$$

for $\xi \in(n-1, n+1)$. Direct computation shows $\left|g_{K}^{\prime \prime}(x)\right| \leq c / x^{3 / 2}$ for $x$ sufficiently large and a constant $c>0$. Therefore, the right-hand side of (4.1) is summable.

Next, since

$$
\frac{b_{n+1}}{a_{n}}-\frac{b_{n}}{a_{n-1}} \frac{\alpha_{n-1}}{\alpha_{n}}=\frac{b_{n+1}-b_{n}}{a_{n}}+\frac{b_{n}}{a_{n-1}}\left(\frac{a_{n-1}}{a_{n}}-\frac{\alpha_{n-1}}{\alpha_{n}}\right),
$$

the condition Theorem 1.1(d) reduces to showing Theorem 1.1(c):

$$
\sum_{n=0}^{\infty} \frac{1}{a_{n-1}}\left|\frac{a_{n-1}}{a_{n}}-\frac{n-1}{n} \frac{g_{K}(n-1)}{g_{K}(n)} \frac{a_{n}}{a_{n-1}}\right|<\infty .
$$

For constants $K^{\prime}$ and $c>0$, we have

$$
\frac{a_{n-1}}{a_{n}}-\frac{n-1}{n} \frac{g_{K}(n-1)}{g_{K}(n)} \frac{a_{n}}{a_{n-1}} \geq \frac{1}{1+\frac{K^{\prime}}{n}+c_{n}}-\left(1-\frac{1}{n}\right)\left(1+\frac{K^{\prime}}{n}+c_{n}\right) \geq-\frac{c}{n}-c_{n}^{\prime}
$$

for a summable sequence $c_{n}^{\prime}$. On the other hand,

$$
\begin{aligned}
\frac{a_{n-1}}{a_{n}}-\frac{n-1}{n} \frac{g_{K}(n-1)}{g_{K}(n)} \frac{a_{n}}{a_{n-1}} & \leq \frac{1}{1-c_{n}}-\left(1-\frac{1}{n}\right) \frac{g_{K}(n-1)}{g_{K}(n)}\left(1-c_{n}\right) \\
& =1-\frac{g_{K}(n-1)}{g_{K}(n)}+c_{n}^{\prime}=\frac{g_{K}(n)-g_{K}(n-1)}{g_{K}(n)}+c_{n}^{\prime}
\end{aligned}
$$

for a summable sequence $c_{n}^{\prime}$. Hence as previously, Taylor's formula applied to $g_{K}$ at the point $n-1$ gives

$$
\frac{a_{n-1}}{a_{n}}-\frac{n-1}{n} \frac{g_{K}(n-1)}{g_{K}(n)} \frac{a_{n}}{a_{n-1}} \leq \frac{c}{n}+c_{n}^{\prime \prime}
$$


for a constant $c>0$ and summable sequence $c_{n}^{\prime \prime}$. Finally, condition (d) leads to (4.2).

Remark 4.4 When we compare Theorem 4.2 with Theorem 4.3 , we see that Theorem 4.3 is interesting only in the case when $\sum_{n=0}^{\infty} 1 / a_{n}^{2}<\infty$. In this case, the condition Theorem $4.3(\mathrm{~d})$ is satisfied.

A sequence similar to $\alpha_{n}=n a_{n}^{-1}$ was used in the proof of [20, Theorem 4.1] and [14, Theorem 2.1]. There it was shown that under the stronger assumptions (which in particular imply $c_{n} \equiv 0, b_{n} \equiv 0$ and $K=0$ ), the measure $\mu$ is absolutely continuous. Whether $\sigma(A)=\mathbb{R}$ was not investigated.

Example 4.5 Let $K>0$. Fix $M$ such that $\log ^{(K)}(M)>0$. Then for the sequences $a_{n}=(n+M) g_{K}(n+M)$ and $b_{n} \equiv 0$, the assumptions of Theorem 4.3 are satisfied.

\section{Applications of Theorem 4.2}

\subsection{Birth and Death Processes}

Given sequences $\left\{\lambda_{n}\right\}_{n=0}^{\infty}$ and $\left\{\mu_{n}\right\}_{n=0}^{\infty}$ such that $\lambda_{n}>0, \mu_{n+1}>0(n \geq 0)$ and $\mu_{0} \geq 0$, we set

$$
Q=\left(\begin{array}{ccccc}
-\left(\lambda_{0}+\mu_{0}\right) & \lambda_{0} & 0 & 0 & \ldots \\
\mu_{1} & -\left(\lambda_{1}+\mu_{1}\right) & \lambda_{1} & 0 & \ldots \\
0 & \mu_{2} & -\left(\lambda_{2}+\mu_{2}\right) & \lambda_{2} & \ldots \\
0 & 0 & \mu_{3} & -\left(\lambda_{3}+\mu_{3}\right) & \ldots \\
\vdots & \vdots & \vdots & \vdots & \ddots
\end{array}\right)
$$

Let us define

$$
\ell^{2}(\pi)=\left\{x \in \mathbb{C}^{\mathbb{N}}: \sum_{n=0}^{\infty} \pi_{n}\left|x_{n}\right|^{2}<\infty\right\}, \quad\langle x, y\rangle_{\ell^{2}(\pi)}=\sum_{n=0}^{\infty} \pi_{n} x_{n} \overline{y_{n}}
$$

where

$$
\pi_{0}=1, \quad \pi_{n}=\frac{\lambda_{0} \lambda_{1} \ldots \lambda_{n-1}}{\mu_{1} \mu_{2} \ldots \mu_{n}}
$$

The operator $Q$ is well defined on the domain $\operatorname{Dom}(Q)=\left\{x \in \ell^{2}(\pi): Q x \in\right.$ $\left.\ell^{2}(\pi)\right\}$. Notice that any sequence with finite support belongs to $\operatorname{Dom}(Q)$. If the operator $Q$ is self-adjoint, it is of a probabilistic interest to examine the spectrum $\sigma(Q)$ of the operator $Q$ (see, e.g., [17]).

Theorem 5.1 Let $a=\left(\mu_{1}, \lambda_{1}, \mu_{2}, \lambda_{2}, \mu_{3}, \lambda_{3}, \ldots\right)$. Assume

(a) $\lim _{n \rightarrow \infty} a_{n}=\infty$, 
(b) $\sum_{n=0}^{\infty} \frac{1}{a_{n}}=\infty$,

(c) $\sum_{n=0}^{\infty}\left[\frac{a_{n+1}}{a_{n}}-1\right]^{-}<\infty$.

Then the matrix $Q$ is self-adjoint and satisfies $\sigma_{\mathrm{p}}(Q)=\emptyset$ and $\sigma(Q)=(-\infty, 0]$.

Proof Let $P$ be a diagonal matrix with entries $\sqrt{\pi_{n}}$ on the main diagonal. Then we have $\bar{A}=P Q P^{-1}$, where $\bar{A}$ is the Jacobi matrix associated with sequences $\bar{a}_{n}=\sqrt{\lambda_{n} \mu_{n+1}}$ and $\bar{b}_{n}=-\left(\lambda_{n}+\mu_{n}\right)$ (see [18, Section 2]). Since the matrix $P: \ell^{2}(\pi) \rightarrow \ell^{2}$ is an isometry (hence $P$ and $P^{-1}$ are bounded), it is enough to consider only the spectrum of $\bar{A}$. By virtue of Proposition 2.2, it is sufficient to consider the spectrum of the matrix $\widehat{A}$, corresponding with the sequences $\left\{a_{n}\right\}$ and $\left\{-b_{n}\right\}$.

Let us consider the case $\mu_{0}=0$. Let $\widetilde{b}_{n} \equiv 0$ and

$$
\widetilde{a}=\left(\sqrt{\lambda_{0}}, \sqrt{\mu_{1}}, \sqrt{\lambda_{1}}, \sqrt{\mu_{2}}, \sqrt{\lambda_{2}}, \ldots\right) .
$$

Observe that by Proposition 2.3 , we have $\widetilde{A}_{e}=\widehat{A}$. Hence, by Theorem 4.2 , the conclusion follows.

Next, suppose that $\mu_{0}>0$. Let $\widetilde{b}_{n} \equiv 0$ and

$$
\widetilde{a}=\left(\sqrt{\mu_{0}}, \sqrt{\lambda_{0}}, \sqrt{\mu_{1}}, \sqrt{\lambda_{1}}, \sqrt{\mu_{2}}, \sqrt{\lambda_{2}}, \ldots\right) .
$$

Applying Proposition 2.3 to $\widetilde{A}_{o}=\widehat{A}$ by Theorem 4.2, we finish the proof.

In [16] it was shown that in the case when $\lambda_{n}=\mu_{n+1}=n+a, a>0$, the matrix $Q$ has purely absolutely continuous spectrum and $\sigma(Q)=(-\infty, 0]$. Theorem 5.1 is applicable in more general situations.

In [21], the following conjecture about spectral properties of operators of the form (5.1) was stated.

Conjecture 5.2 (Roehner and Valent [21]) Assume that

$$
\lim _{n \rightarrow \infty} \mu_{n} / \lambda_{n}=1, \quad \lim _{n \rightarrow \infty} \lambda_{n} / n^{\alpha}=a
$$

for constants $a>0$ and $0<\alpha \leq 2$. Then $\sigma_{p}(Q)=\emptyset$.

In [3] it was shown that without additional assumptions, the conjecture is false. In Theorem 5.1, we provide sufficient conditions when Conjecture 5.2 holds.

It is worthwhile to compare Theorem 5.1 with results obtained in [18]. Let

$$
\lim _{n \rightarrow \infty} \mu_{n} / \lambda_{n}=q \quad(0<q<\infty) .
$$

Then in [18], it was concluded that under additional assumptions (which in particular imply $\lambda_{k+1} / \lambda_{k} \rightarrow 1, \mu_{k+1} / \mu_{k} \rightarrow 1, \lambda_{k} \rightarrow \infty$ and $\alpha<1$ ), the matrix Q satisfies 
$\sigma_{\text {ess }}(Q)=\emptyset$. However, there is a problem in the proof of Lemma 1(iii) on the page 69 . The author states that $\left\|F D^{-1}\right\|_{\ell^{2}}<1$ if for a certain $\zeta>0$,

$$
\frac{\sqrt{\lambda_{k} \mu_{k+1}}}{\lambda_{k}+\mu_{k}+\zeta}<\frac{1}{2}, \quad \frac{\sqrt{\lambda_{k} \mu_{k+1}}}{\lambda_{k+1}+\mu_{k+1}+\zeta}<\frac{1}{2} .
$$

In fact what we need is

$$
\frac{\sqrt{\lambda_{k} \mu_{k+1}}}{\lambda_{k}+\mu_{k}}<\frac{1}{2}-\epsilon, \quad \frac{\sqrt{\lambda_{k} \mu_{k+1}}}{\lambda_{k+1}+\mu_{k+1}}<\frac{1}{2}-\epsilon
$$

for a certain $\epsilon>0$, which under the assumption $q=1$ is impossible because the left-hand sides converge to $1 / 2$. In fact Theorem 5.1 implies the opposite conclusion to results from [18].

Note that

$$
\lim _{n \rightarrow \infty} \frac{\lambda_{n} \mu_{n+1}}{\left(\lambda_{n}+\mu_{n}\right)\left(\lambda_{n+1}+\mu_{n+1}\right)}=\frac{q}{(1+q)^{2}},
$$

which under the assumption $q \neq 1$ is strictly less than $1 / 4$. Therefore [3, Theorem 1] (for a functional analytic proof see [24, Theorem 2.6]) combined with Proposition 2.2 implies that if the matrix $Q$ is self-adjoint and $\lambda_{k} \rightarrow \infty$, then $\sigma_{\text {ess }}(Q)=\varnothing$.

\subsection{Chihara's Problem}

In [1] (see also [2, IV-Theorem 4.2]), the following result was proved.

Theorem 5.3 (Chihara [1] ) Assume that a Jacobi matrix A is self-adjoint, $b_{n} \rightarrow \infty$, the smallest point $\rho$ of $\sigma_{\text {ess }}(A)$ is finite, and

$$
\lim _{n \rightarrow \infty} \frac{a_{n}^{2}}{b_{n} b_{n+1}}=\frac{1}{4}
$$

Then the set $\left\{x: p_{n}(x)=0, n \in \mathbb{N}\right\}$ of the zeros of orthogonal polynomials $\left\{p_{n}\right\}$ is dense in $[\rho, \infty)$.

This suggests the following problem stated in [4] and [5].

Problem 5.4 (Chihara [4,5]) Let the assumptions of Theorem 5.3 be satisfied. Find additional assumptions (if needed) to assure that $\sigma_{\mathrm{ess}}(A)=[\rho, \infty)$.

The following theorem gives sufficient (and easy to verify) additional conditions to Problem 5.4. In fact every Jacobi matrix with $b_{n} \equiv 0$ and $a_{n+1} / a_{n} \rightarrow 1$ from this article provides an example (via Proposition 2.3) satisfying the conclusion of Problem 5.4.

\section{Theorem 5.5 Assume}

(a) $\lim _{n \rightarrow \infty} a_{n}=\infty$, 
(b) $\sum_{n=0}^{\infty} \frac{1}{a_{n}}=\infty$,

(c) $\sum_{n=0}^{\infty}\left[\frac{a_{n+1}}{a_{n}}-1\right]^{-}<\infty$,

(d) $\lim _{n \rightarrow \infty}\left[a_{n-1}-b_{n}+a_{n}\right]=M$.

Then the Jacobi matrix A satisfies $\sigma_{\mathrm{ess}}(A)=[-M, \infty)$. Moreover, if $a_{n+1} / a_{n} \rightarrow 1$, then

$$
\lim _{n \rightarrow \infty} \frac{a_{n}^{2}}{b_{n} b_{n+1}}=\frac{1}{4} .
$$

Proof We show (5.2) by a direct computation. Without loss of generality, we may assume that $M=0$. Let $-r_{n}=a_{n-1}-b_{n}+a_{n}$. Then $a_{n-1}-\left(b_{n}-r_{n}\right)+a_{n}=0$. Let $\widetilde{A}$ be the Jacobi matrix for sequences $\widetilde{a}_{n}=a_{n}, \widetilde{b}_{n}=b_{n}-r_{n}$. The matrix $R=A-\widetilde{A}$ defines a compact self-adjoint operator on $\ell^{2}$ (because $r_{n} \rightarrow 0$ ). Hence, by the Weyl perturbation theorem (see [25]), $\sigma_{\text {ess }}(A)=\sigma_{\text {ess }}(\widetilde{A})$. Theorem 5.1 implies that $\sigma_{\text {ess }}(\widetilde{A})=(-\infty, 0]$. Finally, Proposition 2.2 applied to the matrix $\widetilde{A}$ finishes the proof.

\section{Examples}

Example 6.1 Let $b_{n} \equiv 0, \epsilon>0, a_{0}=\epsilon$, and $a_{2 k-1}=a_{2 k}=\widetilde{a}_{k}(k \geq 1)$ for a sequence $\widetilde{a}_{k}, \widetilde{a}_{k} \rightarrow \infty$. Then the matrix $A$ is always self-adjoint. Moreover, 0 is its eigenvalue if and only if

$$
\sum_{k=0}^{\infty}\left(\frac{a_{0} a_{2} \ldots a_{2 k}}{a_{1} a_{3} \ldots a_{2 k+1}}\right)^{2}=\epsilon^{2} \sum_{k=1}^{\infty} \frac{1}{\widetilde{a}_{k}^{2}}<\infty,
$$

(see, e.g., [12, Theorem3.2]). Therefore the condition Theorem 4.2(b) could not be weakened even for the class of monotonic sequences $a_{n}$.

In [19] it was shown that for $\widetilde{a}_{k}=k^{\alpha},(\alpha \in(0,1))$ the spectrum $\sigma(C)=\mathbb{R}$. In the case $\alpha \leq 1 / 2$, the measure $\mu(\cdot)=\left\langle E(\cdot) \delta_{0}, \delta_{0}\right\rangle$ is absolutely continuous, whereas for $\alpha>1 / 2$, the measure $\mu$ is absolutely continuous on the set $\mathbb{R} \backslash\{0\}$.

Example 6.2 Let $b_{n} \equiv 0$ and $a_{n}=n^{\alpha}+c_{n}(0<\alpha \leq 2 / 3)$, where $c_{2 n}=1$ and $c_{2 n+1}=0$. Then (see [9]) $\sigma(A)=\mathbb{R} \backslash(-1,1)$, and the measure $\mu$ is absolutely continuous on $\mathbb{R} \backslash[-1,1]$. It shows that the condition Theorem 4.2 (c) could not be replaced by $\left[\left(a_{n+1} / a_{n}\right)^{2}-1\right]^{-} \rightarrow 0$.

Example 6.3 Let $a_{0}=1$. For $k ! \leq n<(k+1)$ !, we define $a_{n}=\sqrt{k !}$. For $n>0$, we have

$$
\frac{a_{n+1}}{a_{n}}= \begin{cases}\sqrt{k} & \text { if } n+1=k ! \\ 1 & \text { otherwise }\end{cases}
$$


Define $b_{n} \equiv 0$. We have $a_{n} \leq \sqrt{n+1}$. Therefore $\sum_{n=0}^{\infty} 1 / a_{n}^{2}=\infty$. Observe that the assumptions of Theorem 4.2 are satisfied. Moreover, $a_{n+1} / a_{n} \nrightarrow 1$ and neither [15, Theorem 3.1] nor [11, Lemma 2.6] can be applied.

Acknowledgments The author would like to thank Ryszard Szwarc and Bartosz Trojan for their helpful suggestions concerning the presentation of this article.

Open Access This article is distributed under the terms of the Creative Commons Attribution 4.0 International License (http://creativecommons.org/licenses/by/4.0/), which permits unrestricted use, distribution, and reproduction in any medium, provided you give appropriate credit to the original author(s) and the source, provide a link to the Creative Commons license, and indicate if changes were made.

\section{References}

1. Chihara, T.S.: Orthogonal polynomials whose zeros are dense in intervals. J. Math. Anal. Appl. 24, 362-371 (1968)

2. Chihara, T.S.: An introduction to orthogonal polynomials. In: Mathematics and its Applications, vol. 13, pp. 1-249. Gordon and Breach Science Publishers, New York-London-Paris (1978)

3. Chihara, T.S.: On the spectra of certain birth and death processes. SIAM J. Appl. Math. 47(3), 662-669 (1987)

4. Chihara, T.S.: The one-quarter class of orthogonal polynomials. Rocky Mt. J. Math. 21(1), 121-137 (1991)

5. Chihara, T.S.: An analog of the Blumenthal-Nevai theorem for unbounded intervals. J. Comput. Appl. Math. 153(1-2), 535-536 (2003)

6. Clark, S.L.: A spectral analysis for self-adjoint operators generated by a class of second order difference equations. J. Math. Anal. Appl. 197(1), 267-285 (1996)

7. Dombrowski, J.: Tridiagonal matrix representations of cyclic selfadjoint operators. II. Pac. J. Math. 120(1), 47-53 (1985)

8. Dombrowski, J.: Cyclic operators, commutators, and absolutely continuous measures. Proc. Am. Math. Soc. 100(3), 457-463 (1987)

9. Dombrowski, J., Janas, J., Moszyński, M., Pedersen, S.: Spectral gaps resulting from periodic perturbations of a class of Jacobi operators. Constr. Approx. 20(4), 585-601 (2004)

10. Dombrowski, J., Nevai, P.: Orthogonal polynomials, measures and recurrence relations. SIAM J. Math. Anal. 17(3), 752-759 (1986)

11. Dombrowski, J., Pedersen, S.: Spectral measures and Jacobi matrices related to Laguerre-type systems of orthogonal polynomials. Constr. Approx. 13(3), 421-433 (1997)

12. Dombrowski, J., Pedersen, S.: Absolute continuity for unbounded Jacobi matrices with constant row sums. J. Math. Anal. Appl. 267(2), 695-713 (2002)

13. Dombrowski, J., Pedersen, S.: Spectral transition parameters for a class of Jacobi matrices. Stud. Math. 152(3), 217-229 (2002)

14. Janas, J., Moszyński, M.: Alternative approaches to the absolute continuity of Jacobi matrices with monotonic weights. Integral Equ. Oper. Theory 43(4), 397-416 (2002)

15. Janas, J., Naboko, S.: Multithreshold spectral phase transitions for a class of Jacobi matrices. Oper. Theory Adv. Appl. 124, 267-285 (2001)

16. Janas, J., Naboko, S.: Spectral properties of selfadjoint Jacobi matrices coming from birth and death processes. Oper. Theory Adv. Appl. 127, 387-397 (2001)

17. Karlin, S., McGregor, J.L.: The differential equations of birth-and-death processes, and the Stieltjes moment problem. Trans. Am. Math. Soc. 85, 489-546 (1957)

18. Kreer, M.: Analytic birth-death processes: a Hilbert-space approach. Stoch. Process. Appl. 49(1), 65-74 (1994)

19. Moszyński, M.: Spectral properties of some Jacobi matrices with double weights. J. Math. Anal. Appl. 280(2), 400-412 (2003)

20. Pedersen, S.: Absolutely continuous Jacobi operators. Proc. Am. Math. Soc. 130(8), 2369-2376 (2002). (electronic) 
21. Roehner, B., Valent, G.: Solving the birth and death processes with quadratic asymptotically symmetric transition rates. SIAM J. Appl. Math. 42(5), 1020-1046 (1982)

22. Sahbani, J.: Spectral theory of certain unbounded Jacobi matrices. J. Math. Anal. Appl. 342(1), 663-681 (2008)

23. Simon, B.: The classical moment problem as a self-adjoint finite difference operator. Adv. Math. 137(1), 82-203 (1998)

24. Szwarc, R.: Absolute continuity of spectral measure for certain unbounded Jacobimatrices. In: Buhmann, M.D., Mache, D.H. (eds.) Advanced Problems in Constructive Approximation, ISNM International Series of Numerical Mathematics, vol. 142, pp. 255-262. Birkhäuser, Basel (2003). doi:10. 1007/978-3-0348-7600-1_18

25. Weyl, H.: Über beschränkte quadratische Formen, deren Differenz vollstetig ist. Rend. Circ. Mat. Palermo 27, 373-392 (1909) 